\title{
Cryogenic resonator design for trapped ion experiments in Paul traps
}

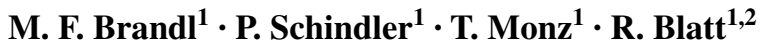

Received: 28 January 2016 / Accepted: 28 April 2016 / Published online: 18 May 2016

(c) The Author(s) 2016. This article is published with open access at Springerlink.com

\begin{abstract}
Trapping ions in Paul traps require high radio frequency voltages, which are generated using resonators. When operating traps in a cryogenic environment, an invacuum resonator showing low loss is crucial to limit the thermal load to the cryostat. In this study, we present a guide for the design and production of compact, shielded cryogenic resonators. We produced and characterized three different types of resonators and furthermore demonstrate efficient impedance matching of these resonators at cryogenic temperatures.
\end{abstract}

\section{Introduction}

Over the last two decades, the application of ion traps has expanded from mass spectrometry [1] and frequency standards $[2,3]$ toward engineering of quantum systems which can be used for quantum computation [4-6] and quantum simulation $[7,8]$. It is commonly accepted that largescale trapped ion quantum information processors require micrometer-scale ion traps [5, 9]. Such traps usually suffer from excessive electric field noise close to metallic surfaces at room temperature, but at cryogenic temperatures, this noise is strongly reduced $[10,11]$. Thus, it seems natural to move toward cryogenic experimental setups which have the additional advantage that the ambient pressure is usually a

M. F. Brandl

Matthias.Brandl@uibk.ac.at

1 Institut für Experimentalphysik, Universität Innsbruck, Technikerstraße 25, 6020 Innsbruck, Austria

2 Institut für Quantenoptik und Quanteninformation der Österreichischen Akademie der Wissenschaften, Technikerstraße 21a, 6020 Innsbruck, Austria few orders of magnitude lower than at room temperature. This enables trapping of longer ion strings due to fewer collisions with background gas. Furthermore, no bake-out procedure of the vacuum vessel is required, allowing for rapid trap cycle times.

The operation of Paul traps requires high radio frequency (RF) voltages, which are usually generated with the aid of the voltage gain present in RF resonators. For this purpose, helical resonators are typically used in the frequency regime up to $50 \mathrm{MHz}$, whereas for experiments requiring higher drive frequencies coaxial resonators have been used as well [12-14]. In cryogenic experiments, the resonator needs to fulfill different criteria than in room temperature experiments where the resonator can be placed outside the vacuum vessel. In particular, the connections in a cryostat need to have low thermal conductivity to limit the thermal load. Following the Wiedemann-Franz law [15], these results in a low electrical conductivity between room temperature and the cryogenic parts of the experiment. Thus, the resonators have to be operated at the cold stage. Moreover, space constraints are stricter in cryogenic systems which makes helical resonators undesirable as they are generally bulky ${ }^{1}$. To minimize the volume of the resonator, an RLC series resonator can be used [17].

In Sect. 2, we focus on the choice of the trap drive frequency, the required voltage gain, and voltage monitoring. Section 3 covers coil design for three types of coils, and in the following Sect. 4, we discuss impedance matching of the resonator and present an efficient way to match cryogenic resonators. Section 5 focuses on the design of RF shielding, and finally, we present the results in Sect. 6.

\footnotetext{
${ }^{1}$ Helical resonators have been used in cryogenic systems [16].
} 


\section{General considerations}

In trapped ion experiments, RF voltages of several hundreds of volts are commonly applied to the trap, which has a simple capacitor as its electrical circuit equivalent. Thus, we need to design a resonator that maximizes the RF voltage at this capacitor for a given input power. In this section, we discuss general considerations in resonator design which are not limited to a specific resonator type.

\subsection{Choosing the trap drive frequency}

At first, we consider the losses in an RLC resonator and its scaling with frequency. From the solution of the equation of motion of a trapped ion in a Paul trap (Mathieu equation), one obtains the trap voltages with the stability parameter $q$ [18], which scales as

$q=\frac{2 e V}{M r_{0}^{2} \Omega^{2}} \propto \frac{V}{\Omega^{2}}$

where $V$ is the amplitude of the RF voltage at the trap and $\Omega$ is the trap drive (angular) frequency. Hence, for constant $q$, the loss power scales as

$P \propto V^{2} \propto \Omega^{4}$.

A low trap drive frequency will reduce the losses. On the other hand, high secular motion frequencies of the ion in the trap are desirable for operation. Higher secular frequencies require a higher $\Omega$, and thus, the desired secular frequencies set a lower limit for $\Omega$. In this study, we aim for a $q$ around 0.25 , an axial secular frequency of $1 \mathrm{MHz}$, and both radial frequencies to be $3.7 \mathrm{MHz}$. This will require a trap drive frequency of $\Omega=2 \pi 42.6 \mathrm{MHz}$. Simulations of our trap show that we need a drive voltage of about $170 V_{\mathrm{rms}}$ to reach the desired trap frequencies. In order to limit the thermal load onto the cryostat from dissipated power in the resonator, this voltage should be reached with less than $100 \mathrm{~mW}$ of $\mathrm{RF}$ input power. This power corresponds to a consumption of about 1/7 1 of liquid helium per hour when operating a wet cryostat at $4.2 \mathrm{~K}[19]$.

\subsection{Voltage gain of an RLC resonator}

Figure 1 shows an RLC series resonator driven through a matching network. In the following, we will assume perfect impedance matching with a loss-free matching network and that the losses in the connecting cable do not influence the resonator circuit. Thus, we can set the input power equal to the loss power in the resonator $P_{\text {input }}=P_{\text {loss,resonator, }}$, where $P_{\text {input }}=\frac{V_{\text {in }}^{2}}{R_{\text {wave }}}$ with $V_{\text {in }}$ being the input voltage supplied to the circuit and $R_{\text {wave }}$ the wave impedance of the connecting cable, commonly $50 \Omega$. We can further write

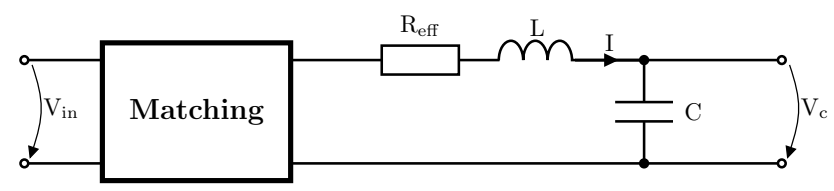

Fig. 1 An RLC series resonator with a matching network used to generate high RF voltages at the trap

$P_{\text {loss,resonator }}=|I|^{2} \cdot R_{\text {eff }}=\left|\frac{V_{\mathrm{c}}}{\frac{1}{i \Omega C}}\right|^{2} \cdot R_{\text {eff }}$,

where $I$ is the current in the resonator, $R_{\text {eff }}$ its effective loss resistance, $\Omega$ its frequency, $V_{\mathrm{c}}$ the voltage at the capacitor, and $C$ the capacitor representing the trap. Thus, we obtain

$\frac{V_{\text {in }}^{2}}{R_{\text {wave }}}=\left|\Omega C V_{\text {in }} G_{\mathrm{V}}\right|^{2} \cdot R_{\text {eff }}$

where $G_{\mathrm{V}}$ is the voltage gain of the circuit, defined as $G_{\mathrm{V}}=\frac{V_{\mathrm{c}}}{V_{\text {in }}}$.

The quality factor $Q$ of a resonator is defined as the resonance frequency $f_{0}$ divided by the bandwidth $\Delta f$

$Q=\frac{f_{0}}{\Delta f}$.

In trapped ion experiments, we are usually not interested in a small bandwidth around $\Omega$ but rather in a large voltage gain at the trap drive frequency. It should be noted that the voltage gain and bandwidth are qualitatively but not necessary quantitatively the same for different types of resonators. The quantity that is of direct interest for our application is the voltage gain, which can be derived from (4) as

$G_{\mathrm{V}}=\frac{1}{\Omega \cdot C} \cdot \frac{1}{\sqrt{R_{\text {wave }} \cdot R_{\text {eff }}}}=\sqrt{\frac{Q}{R_{\text {wave }} \cdot \Omega \cdot C}}$

with the quality factor of an ideal RLC resonator $Q=\frac{1}{R_{\mathrm{eff}} \cdot \Omega \cdot C}$. This indicates that both, the capacitive load and the effective resistance of the resonator. should be minimized.

\subsection{Pickup}

Voltage pickups are not required for the operation of the trap, but are useful to measure the voltage on the trap and are required to actively stabilize the RF voltage on the trap. In this section, we discuss inductive and capacitive pickups, as shown in Fig. 2.

A capacitive pickup consists of a voltage divider parallel to the trap, where $C_{\text {pickup2 }} \gg C_{\text {pickup1, which leads to the }}$ pickup voltage 


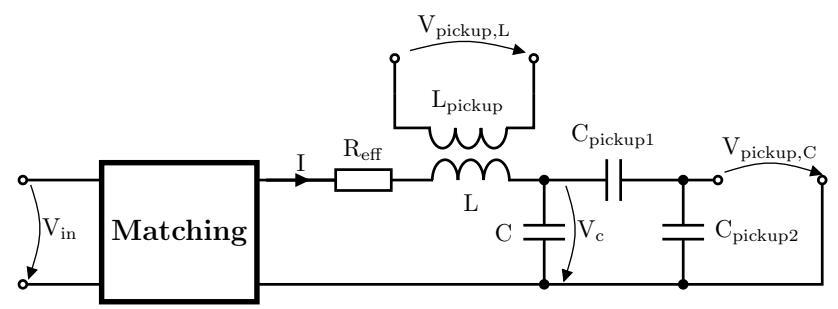

Fig. 2 The circuit of an RLC series resonator with an inductive and a capacitive pickups

$V_{\text {pickup,C }}=\frac{C_{\text {pickup1 }}}{C_{\text {pickup } 1}+C_{\text {pickup2 }}} \cdot V_{\mathrm{c}}$.

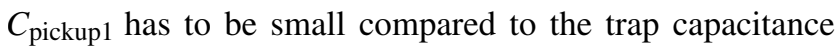
$C$, or the capacitive load of the RLC resonator will increase significantly, reducing $G_{\mathrm{V}}$ as shown in (6). Typical values for $C_{\text {pickup2 }}$ are several hundred times the value of $C_{\text {pickup } 1}$.

In an ideal RLC series resonator, the voltage on the coil is the same as the voltage at the capacitor but with a $180^{\circ}$ phase shift. We can monitor the voltage at the coil as a signal proportional to the voltage at the trap since we are only interested in the amplitude of the trap voltage. Here, $L$ and $L_{\text {pickup }}$ are coupled, and the pickup voltage can be estimated with the derivations from ref. [13].

In order to maintain low losses, the pickup should not add significant losses in the resonator. If one uses a coaxial cable with a wave impedance $R_{\text {wave }}$ to monitor the pickup signal, a resistor $R_{\text {wave }}$ is used as a termination to avoid reflections. The losses are then $V_{\text {pickup }}^{2} / R_{\text {wave }}$ which need to be small compared to the losses in the resonator $P$.

In general, we recommend using an inductive pickup, because it does not increase the capacitive load, which would reduce the voltage gain. However, for experiments, which frequently test different traps, a capacitive pickup may be preferable since the more accurate ratio between pickup and applied voltage facilitates estimating trapping parameters.

\section{Coil design}

Surface traps for cryogenic environments typically use a high-quality dielectric carrier material, and thus, the losses in the resonator are usually dominated by the coil. In this study, we demonstrate the design and production of compact and low-loss coils that should allow us to drive our resonator under the conditions stated in Sect. 2.1. Coils produced with machines are desirable because the production process is reproducible. Furthermore, we prefer toroid coils as they guide the magnetic field in their center, making them less sensitive to their environment. In our application, ferromagnetic core materials are undesirable, because they would induce spatial inhomogeneities of the magnetic field near the trap. We estimate the load capacitance of our resonator to be $10 \mathrm{pF}$, which at a given resonance frequency of $42.6 \mathrm{MHz}$ yields a required inductance of $1.4 \mu \mathrm{H}$.

\subsection{PCB Coils}

Kamby and coworkers [20] demonstrated the integration of toroidal RF inductors into printed circuit boards (PCB). These coils can be fully produced by machines, and in this study, we will refer to these inductors as PCB coils.

Ref. [20] provides specific formulas for the inductance $L$, the resistance of one segment $R_{\mathrm{seg}}$, and the resistance of one via $R_{\text {via }}$ of PCB coils. In order to minimize the losses of the coil, we want to minimize the resistance of one winding while maintaining a constant cross section to keep the inductance constant. The resistance of one winding is defined by

$$
\begin{aligned}
2 R_{\text {seg }}+2 R_{\text {via }} & =2 \frac{R_{\text {seg }}}{r_{\mathrm{o}}-r_{\mathrm{i}}}\left(r_{\mathrm{o}}-r_{\mathrm{i}}\right)+2 \frac{R_{\mathrm{via}}}{h} h \\
& =2 R_{\mathrm{seg}}^{\prime}\left(r_{\mathrm{o}}-r_{\mathrm{i}}\right)+2 R_{\mathrm{via}}^{\prime} h,
\end{aligned}
$$

where $r_{\mathrm{O}}$ and $r_{\mathrm{i}}$ are the outer and the inner radius of the PCB coil, $h$ is the thickness of the PCB, and $R_{\text {seg }}^{\prime}$ and $R_{\text {via }}^{\prime}$ are the average length-dependent resistances of a segment and a via. $R_{\text {seg }}^{\prime}$ and $R_{\text {via }}^{\prime}$ depend on the exact geometry, but usually vias have similar cross sections as the segments making their length-dependent resistances approximately equal. That means, in order to minimize (8) and to keep the cross section of the coil constant, $\left(r_{\mathrm{o}}-r_{\mathrm{i}}\right)$ and $h$ should be similar in size.

We chose Rogers 4350B as the substrate material, because it has a small loss tangent, a similar thermal expansion coefficient as copper, and is ultra-high vacuum compatible. The thickest available Rogers 4350B had $3.1 \mathrm{~mm}$ thickness, which is unfortunately too thin to minimize the resistance for a given cross section.

We produced this type of coil in-house with dimensions obtained from simulations with the formulas from ref. [20]. During multiple iterations, we varied the size and the number of vias. The lowest losses for our production process could be achieved with the design depicted in Fig. 3. The PCB coil has an outer diameter of $35 \mathrm{~mm}$ and an inner diameter $20.4 \mathrm{~mm}$ with a substrate thickness of $3.1 \mathrm{~mm}$. Its 64 windings result in an inductance of $1.4 \mu \mathrm{H}$. We added one winding which is galvanically isolated from the rest of the coil. This winding acts as an inductive pickup and should thus be as reproducible as the entire coil.

\subsection{Wire coils}

For the second type of coils, wire coils, we wound a single wire around a rigid structure leading to a similar 


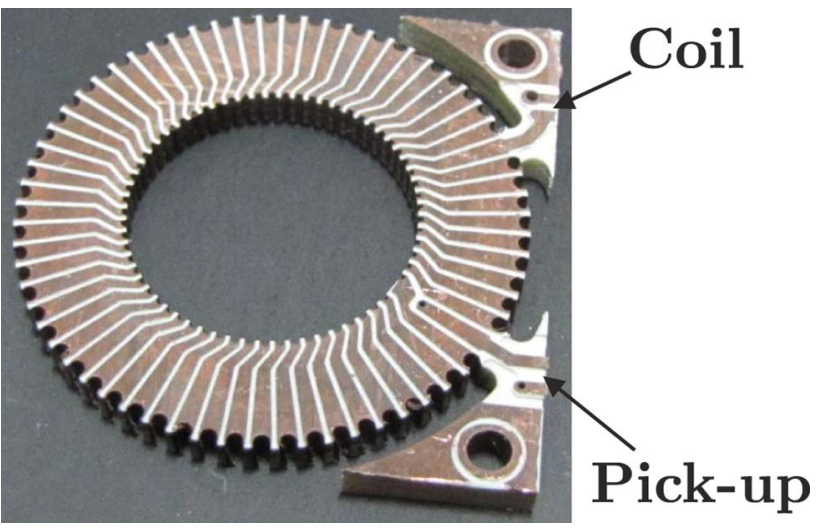

Fig. 3 PCB coil produced in copper with a diameter of $35 \mathrm{~mm}$ and 64 windings. Additionally, there is a pickup coil included in the design with just one winding

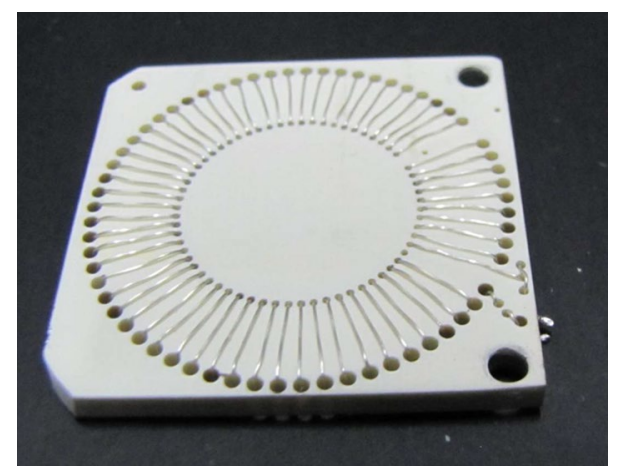

Fig. 4 A wire coil made out of $0.4 \mathrm{~mm}$ thick silver-plated copper wire on a $3.1 \mathrm{~mm}$ thick Rogers $4350 \mathrm{~B}$ core

geometry as the PCB coils. Such a coil is less reproducible than a PCB coil since the wire length will vary for each coil. Hence, its inductance and its resistance will vary as well. A coil with the same geometry as for the PCB coils above, made out of silver-plated copper on a Rogers 4350B substrate, is shown in Fig. 4.

In wire coils, the cross section of the core of a wire coil is bigger than that of a PCB coil, because the wire bends are less sharp. Hence, we expect the inductance of a wire coil to be higher than the one of a PCB coil with the same nominal geometry. The $0.4 \mathrm{~mm}$ thick silver-plated copper wire, which we used for the wire coil, had a cross section similar to the average cross section of a PCB coil. Therefore, we expect similar losses in both types of coils with the same geometries. These considerations allow us to characterize the production processes of the PCB coils by comparing the two types of coils. Higher losses in the PCB coils are an indication that the production of the PCB coils can be improved.

Since we do not need traces on the substrate, it is possible to use any machinable material with a low-loss tangent. We also produced wire coils with a Teflon core since it has

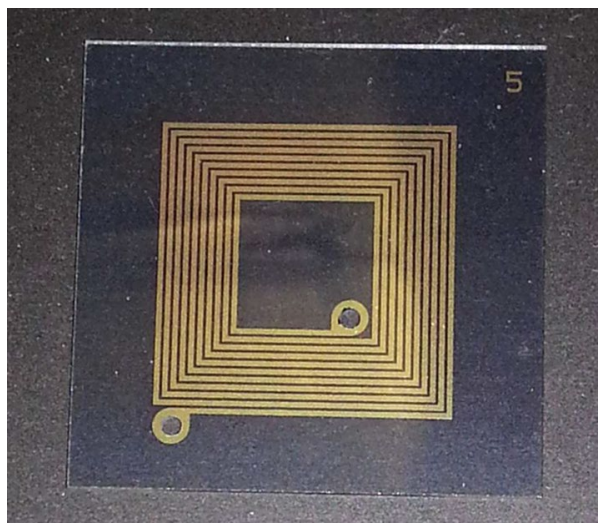

Fig. 5 An yttrium barium copper oxide (YBCO) coil with a $200 \mathrm{~nm}$ protection layer of gold on a $25 \mathrm{~mm} \times 25 \mathrm{~mm}$ piece of sapphire

a lower loss tangent and a lower dielectric constant than Rogers 4350B. Thus, the losses will be less affected by the core material and the self-resonance frequency of the coil will be higher. Another option to minimize the losses in the coil can be to use a superconducting wire to reduce the ohmic losses of the resonator.

\subsection{Spiral coil}

The quality factor of the presented coils is limited by the ohmic resistance of the conductor. Thus, a coil based on a superconductor is expected to result in lower losses in the resonator. Furthermore, it could be necessary to operate the experiment at temperatures above $15 \mathrm{~K}$ to reduce the liquid helium consumption in wet cryostats. At these temperatures, a superconductor with a high critical temperature such as a high-temperature superconductor (HTS) is required. However, a coil made from a readily available HTS material needs to be a two-dimensional (2D) structure due to manufacturing constraints, and we choose to use a spiral coil as shown in Fig. 5.

Since such coils require only 2D structuring, they can be manufactured in a very accurate and reproducible way. For the demonstrated spiral coil, a HTS was produced by Ceraco Ceramic Coating ${ }^{2}$. Our coils were made out of an yttrium barium copper oxide (YBCO) film of $330 \mathrm{~nm}$ thickness on a sapphire wafer. To facilitate soldered connections, a $200 \mathrm{~nm}$ film of gold was placed on top of the superconductor. This film is thinner than the penetration depth due to skin effect around the expected $42.6 \mathrm{MHz}$ and thus should not dominate the losses in the resonator. The critical temperature of this YBCO film is above $87 \mathrm{~K}$, and its critical current density is higher than $2 \cdot 10^{6} \mathrm{~A} / \mathrm{cm}^{2}$ at $77 \mathrm{~K}$. We can derive the peak current in the

\footnotetext{
${ }^{2}$ Ceraco Ceramic Coating GmbH, Rote-Kreuz-Str. 8, D-85737 Ismaning, Germany.
} 
coil from Sect. 2 to be 0.64 A. Hence, traces with a width of more than $100 \mu \mathrm{m}$ are required to stay below the critical current density.

For our square spiral coils, we chose a trace width of $300 \mu \mathrm{m}$, a trace gap of $150 \mu \mathrm{m}$, and 10 windings. We designed 9 different coils varying the outer diameter between 14 and $20 \mathrm{~mm}$. Equation 4.1 of reference [21] gives an estimate for the inductance, which results in target inductances between $1.2 \mu \mathrm{H}$ and $1.6 \mu \mathrm{H}$ for our coils. This design should allow for coils with very high-quality factors already at temperatures accessible with liquid nitrogen instead of liquid helium.

It should be noted here that superconductors are perfect diamagnets, which are spatial inhomogeneities for magnetic fields. One can align the 2D plane of the spiral coil with the spatially homogeneous magnetic field of the experiment to minimize this effect, but one should always keep this in mind.

\section{Impedance matching}

The resonator is connected to the source through a cable with a given wave resistance $R_{\text {wave, }}$ typically $50 \Omega$. The complex voltage reflection coefficient $\underline{r}_{\mathrm{v}}$ at the transition from the cable to the resonator [22] is then

$\underline{r}_{\mathrm{v}}=\frac{\underline{V}_{\text {reflection }}}{\underline{V}_{\text {input }}}=\frac{\underline{Z}_{\text {res }}-R_{\text {wave }}}{\underline{Z}_{\text {res }}+R_{\text {wave }}}$,

where $\underline{V}_{\text {reflection }}$ is the complex reflected voltage, $\underline{V}_{\text {input }}$ is the complex incoming voltage, and $\underline{Z}_{\text {res }}$ is the complex impedance of the resonator ${ }^{3}$. The reflected power coefficient $R_{\mathrm{p}}$ is then just $R_{\mathrm{p}}=\left|\underline{r}_{\mathrm{v}}\right|^{2}$. Since RLC series resonators have a very low impedance on resonance, $\underline{Z}_{\mathrm{res}}=R_{\mathrm{eff}}$, the transition from a $50 \Omega$ cable to the resonator on resonance will reflect almost all the inserted power. In order to minimize the reflection, a properly designed impedance matching network is required. The most commonly used matching network is the $L$ network as it is the simplest, consisting only of two circuit elements.

Figure 6 shows the two possible $L$ networks where the reactance parallel to the resonator transforms the real part of the impedance to be $R_{\text {wave }}$. The resulting imaginary term is small compared to $R_{\text {wave }}$ (for low-loss resonators) and can be compensated with the series reactance.

The resonator's quality factor from (5) can be written as

$Q_{\text {coil }}=\frac{\Omega L}{R_{\text {eff }}}$

for an RLC series resonator. Figure 7 shows the required values for the matching capacitor $C_{\mathrm{M}}$ and inductance $L_{\mathrm{M}}$ as

\footnotetext{
3 The underline denotes a complex quantity.
}
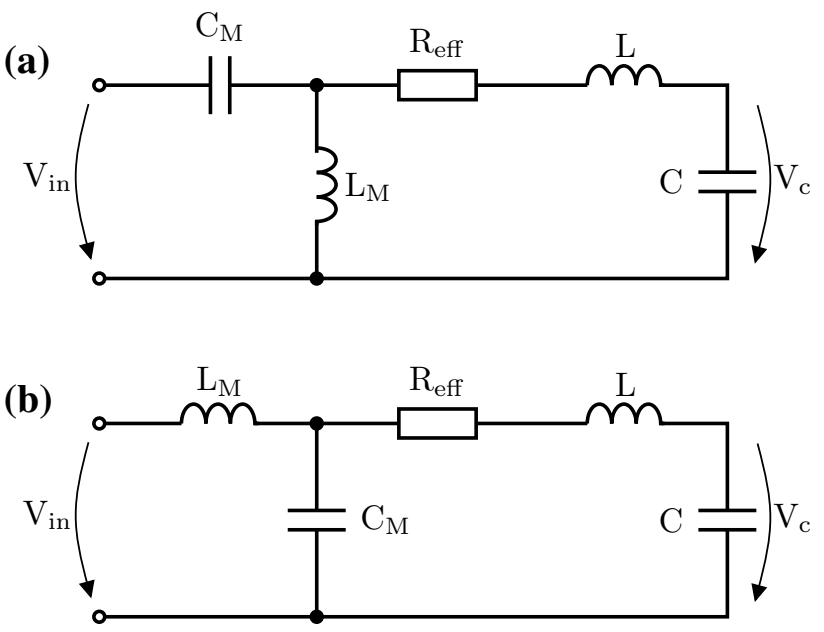

Fig. 6 Two possible matching networks. In a the matching capacitor $C_{\mathrm{M}}$ is in series and the matching coil $L_{\mathrm{M}}$ parallel to the RLC series resonator. In $\mathbf{b}$ the matching coil $L_{\mathrm{M}}$ is in series and the matching capacitor $C_{\mathrm{M}}$ parallel to the RLC series resonator
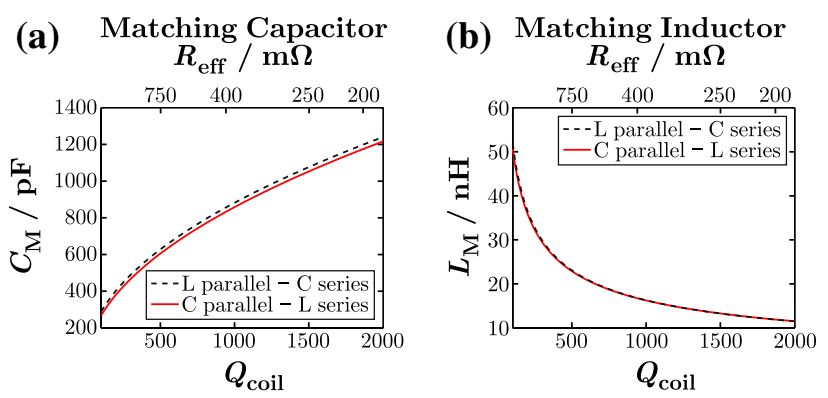

Fig. 7 Calculated values of the matching capacitor $C_{M}$ a and matching coil $L_{\mathrm{M}} \mathbf{b}$ for the matching networks of Fig. 6 over a varying quality factor of the coil. The matching is calculated for a $50 \Omega$ cable, an inductor of $1.4 \mu \mathrm{H}$, and a capacitor of $10 \mathrm{pF}$

a function of the quality factor of the coil with inductance of $1.4 \mu \mathrm{H}$. For quality factors around 1000 , the required inductance $L_{\mathrm{M}}$ is in the range of $10 \mathrm{nH}$. Thin traces on a PCB have approximately a length-dependent inductance of $1 \mathrm{nH} / \mathrm{mm}$. Therefore, small deviations in the trace length will lead to inefficient matching. The strong dependence of the matching on the reactance parallel to the resonator makes the matching network from Fig. 6b more favorable, because capacitances of several hundred $\mathrm{pF}$ can be adjusted with much more precision than inductances of about $10 \mathrm{nH}$. One can incorporate the inductance $L_{\mathrm{M}}$ into the trace on the PCB from the coaxial connector to the matching capacitance, by setting this distance between 1 and $2 \mathrm{~cm}$. Hence, we expect a power reflection of $1 \%$ or less even without adding an additional inductor.

$\mathrm{RF}$ transformers or baluns on the RF input side are commonly used to avoid ground loops between the RF source 


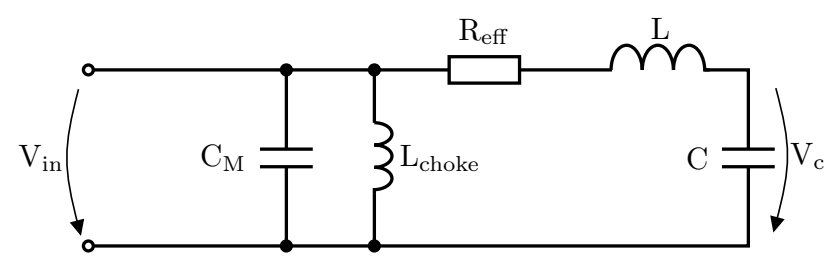

Fig. 8 Chosen matching network with only a matching capacitor $C_{\mathrm{M}}$ parallel to the RLC series resonator. Additionally, one can put an RF choke parallel to $C_{\mathrm{M}}$ to avoid a possible charging up of $C$
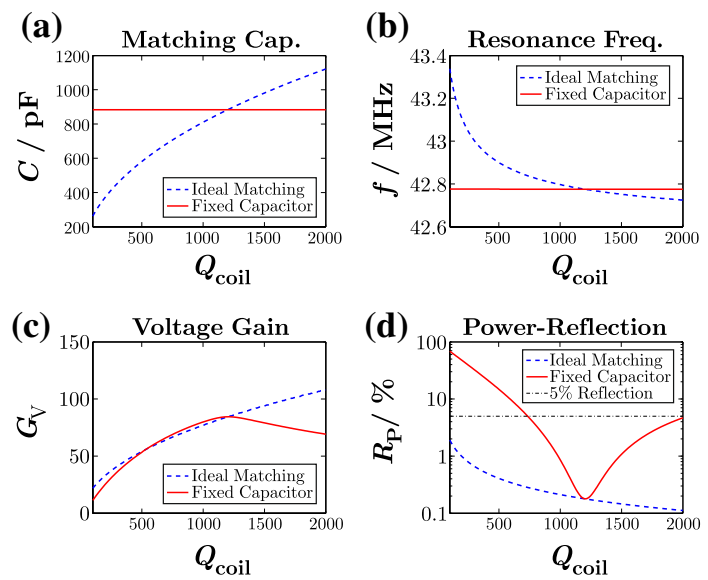

Fig. 9 The matching of these plots is calculated for a $50 \Omega$ cable, an inductor of $1.4 \mu \mathrm{H}$, and a capacitor of $10 \mathrm{pF}$, thus a resonance frequency of $42.6 \mathrm{MHz}$. The red lines show the values for a fixed capacitance $C_{\mathrm{M}}$ chosen for an ideal matching of $Q_{\text {coil }}=1200$. In $\mathbf{a}$, one can see the required matching capacitor as a function of the quality factor of the coil $Q_{\text {coill }}$. b Shows the change in resonance frequency of an ideal match. In $\mathbf{c}$, the voltage gain is plotted over $Q_{\text {coil. }} \mathbf{d}$ Shows the reflection coefficient of the matching network

and the experiment. When using the circuit from Fig. $6 \mathrm{~b}$ in combination with a transformer, the DC potential of the trap electrodes is not defined. We mitigate this by adding an RF choke parallel to the matching capacitor, where $\Omega L_{\text {choke }} \gg \frac{1}{\Omega C_{\mathrm{M}}}$, as depicted in Fig. 8 .

In a cryostat, it is impractical to adjust the matching capacitance $C_{\mathrm{M}}$ during or after a cooldown. Hence, we have to find an efficient way of choosing the best value for $C_{\mathrm{M}}$ with a minimum number of temperature cycles. For this, we simulated our matching circuit for varying and also for a constant value of $C_{\mathrm{M}}$. Fig. 9 shows results of simulations where the fixed capacitor value was chosen for a match at $Q_{\text {coil }}=1200$. Figure 9a depicts an increase in the optimum $C_{\mathrm{M}}$ with increasing $Q_{\text {coil }}$. Figure $9 \mathrm{~b}$ illustrates the dependence of the matched resonance frequency on $Q_{\text {coil }}$. In Fig. 9c, the simulations confirm the dependence of the voltage gain on $Q_{\text {coil }}$, following from (6). If the physical value for $Q_{\text {coil }}$ is smaller than the expected value, used to choose the fixed matching capacitor, the voltage gain is about the

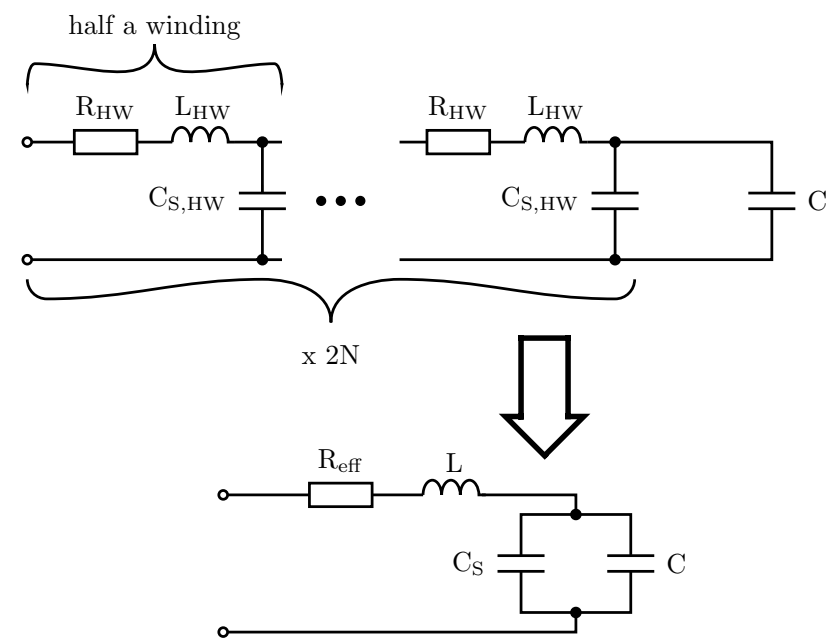

Fig. 10 Model for the capacitance of the RF shield. From the resistance $R_{\mathrm{HW}}$, the inductance $L_{\mathrm{HW}}$, and the capacitance to the shield $C_{\mathrm{S}, \mathrm{HW}}$ of half a winding, one can calculate the total impedance of the circuit, which allows one to derive the total capacitance $C_{\mathrm{S}}$ caused by the shield

same as for an ideal match. But if the actual quality factor $Q_{\text {coil }}$ is higher than the expected value, the voltage gain will be lower than with an ideal match. Hence, underestimating the quality factor of the coil should be avoided since it would result in a significantly lower voltage gain.

Figure $9 \mathrm{~d}$ shows the reflection coefficient as a function of $Q_{\text {coil }}$. The effect of $L_{\mathrm{M}}$ is small, and thus, it was omitted for these simulations. We can see that even without $L_{\mathrm{M}}$, but with an ideally matched $C_{\mathrm{M}}$, a low reflection coefficient can be achieved. If one chooses a constant value for $C_{\mathrm{M}}$ at a match for $Q_{\text {coil }}=1200$, the reflected power will stay below $5 \%$ even if $Q_{\text {coil }}$ varies between 800 and 2000 .

The parameters for exact impedance matching can be estimated with one additional cooling cycle. For the first cycle, one has to guess the value for $Q_{\text {coil }}$ and adjust $C_{\mathrm{M}}$ according to simulations following the approach of Fig. 9a. When the resonator is cold, the reflection coefficient is measured and compared with the simulations in Fig. 9d. The measured reflection coefficient can then only correspond to two values for $Q_{\text {coil }}$. If the reflection coefficient passed a minimum during cooldown, one should choose the higher value, if not, the lower value of $Q_{\text {coil }}$. With this new value for $Q_{\text {coil }}$, one can calculate the correct matching capacitor value $C_{\mathrm{M}}$. Our experience shows that this method will yield a power reflection coefficient of less than $2 \%$ for the second cooldown.

\section{RF shield}

RF resonators emit electromagnetic radiation, which needs to be shielded to minimize unwanted RF interference in 


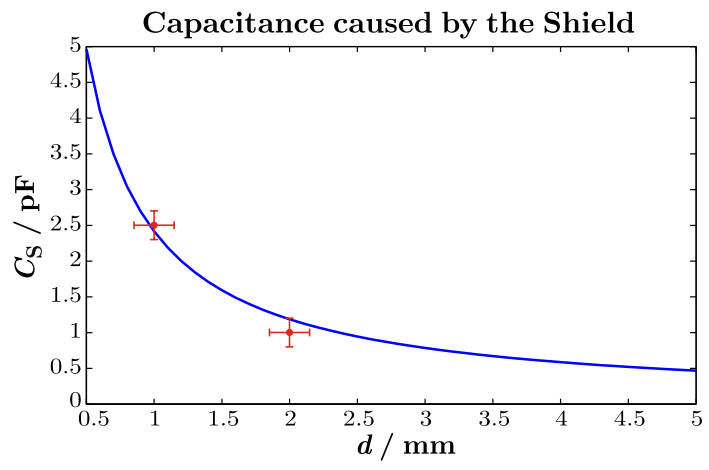

Fig. 11 Simulated capacitance caused by the shield in dependence of the distance between the coil and the shield. The red crosses are measured capacitances for two different shield-coil distances

other parts of the experiment. The RF shield needs to be grounded and thereby generates a capacitance between the resonator and ground. This capacitance has to be minimized as suggested in (6). In order to keep the resonator compact, we have to find a trade-off between the size of the shield and the capacitance added by it.

First, we need to find a model for the shield capacitance $C_{\mathrm{S}}$. Figure 10 depicts a model where we divide the coil in $2 N$ half windings. Each has an ohmic resistance of $R_{\mathrm{HW}}$, an inductance $L_{\mathrm{HW}}=L / 2 N$, and a capacitance to the shield $C_{\mathrm{S}, \mathrm{HW}}$. We can estimate $C_{\mathrm{S}, \mathrm{HW}}$ with the standard formula for a parallel-plate capacitor if we assume that the grounded shield is parallel to the coil. From the calculations of the impedance of the entire circuit, we can extract the effective capacitance $C_{\mathrm{S}}$ that occurs due to the presence of the shield.

The results of these simulations for our PCB coils with an outer radius of $17.5 \mathrm{~mm}$ and an inner radius of $10.2 \mathrm{~mm}$ are shown in Fig. 11. It can be seen that a distance of about $2 \mathrm{~mm}$ is sufficient to keep the additional capacitive load below $1 \mathrm{pF}$. Adapted simulations for the wire coils yield results similar to the ones for the PCB coils. Hence, we can use the same shield for both types of coils.

We expect that the capacitance caused by the shield for the spiral coils is even smaller, because its surface is smaller. Additionally, if we place the spiral coil in the center of the same shield, the distance to the shield will be $1.55 \mathrm{~mm}$ bigger than for the other coils. Hence, the capacitance to ground will decrease further. Moreover, the shield has an influence on the magnetic field lines of the spiral coil in this configuration and thereby also on the inductance and the losses of the coil.

At room temperature, the penetration depth due to skin effect in copper at frequencies around $40 \mathrm{MHz}$ is about $10 \mu \mathrm{m}$. Thus, any mechanically stable shield, of a couple of $1 / 10 \mathrm{~mm}$ thickness, should yield a suitable attenuation. Fig. 12 shows a resonator encased in a silver-plated

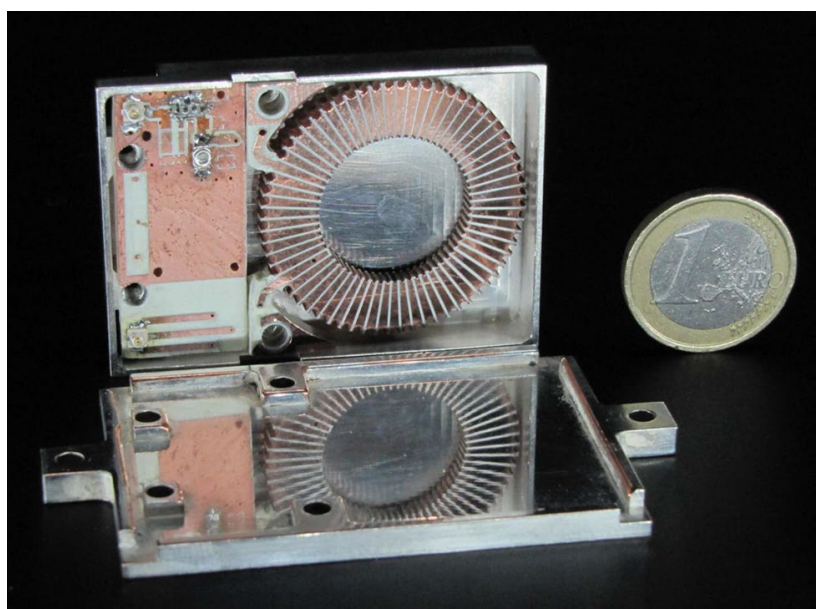

Fig. 12 The RLC resonator built with a PCB coil (right) and the matching network (left) in a silver-plated copper shield. The dimensions of the shielded resonator are $57 \mathrm{~mm} \times 40 \mathrm{~mm} \times 10.2 \mathrm{~mm}$

copper shield. The dimensions of this resonator are $57 \mathrm{~mm} \times 40 \mathrm{~mm} \times 10.2 \mathrm{~mm}$. On the left side of the resonator, one can see a PCB with the matching network at the top and the SMT connector for the inductive pickup at the bottom. On the right side is the PCB coil, and the two PCBs are connected together by soldered joints.

\section{Results}

In our measurement setup, we used a capacitive pickup to measure the voltage at the trap. With the known ratio of this capacitive voltage divider, we could directly measure the voltage at the trap and calibrate the inductive pickup of the coil. All measurements were taken with a test trap as the load capacitance.

Table 1 shows our measurement results. The PCB coils had an inductance very close to the desired value. Unfortunately, we could not achieve the expected quality factor. We had designed the wire coils to have losses similar to the PCB coils, but the PCB coils showed much higher losses than the wire coils. We think this is due to an imperfect production process that we could not improve further with our in-house techniques.

Additionally to the PCB coils in copper, we produced them in silver as well. The inductances were similar, but the losses in the silver coils were even higher. This was unexpected as silver is a better conductor than copper. But the silver coils needed to be reworked after fabrication to remove shorts between the segments. During this process, the silver coating might have been scratched, resulting in higher losses. We expect that silver plating after structuring should reduce the losses compared to a copper coil, but we were unable to do so with our in-house fabrication process. 
Table 1 Measured resonance frequencies, voltage gains, and quality factors of the tested coils

\begin{tabular}{lllll}
\hline Name & $T(\mathrm{~K})$ & $f_{\text {res }}(\mathrm{MHz})$ & $G_{\mathrm{V}}$ & $Q_{\text {coil }}$ \\
\hline PCB coil & 295 & 42.4 & $19(2)$ & $60(13)$ \\
PCB coil & $\sim 80$ & 42.8 & $27(3)$ & $115(27)$ \\
PCB coil & $\sim 10$ & 42.9 & $41(4)$ & $208(43)$ \\
Wire coil & 295 & 36.8 & $25(2)$ & $89(15)$ \\
Wire coil & $\sim 80$ & 37.2 & $36(3)$ & $184(32)$ \\
Wire coil & $\sim 10$ & 37.9 & $58(6)$ & $624(136)$ \\
HTS coil & $<88$ & 44.3 & $92(9)$ & $1172(241)$ \\
Ref. [16] & 4.2 & 13 & 237 & 3000 \\
Ref. [17] & 5.7 & 7.43 & $101(6)$ & $700(30)$ \\
Ref. [23] & 77 & $33-40$ & 20 & - \\
\hline
\end{tabular}

The measurement uncertainties were dominated by the uncertainty in the ratio of this capacitive voltage divider, about $10 \%$

The voltage gain of about 60 of the wire coil on a Rogers 4350B core would require $160 \mathrm{~mW} \mathrm{RF}$ input power to get to the desired $170 V_{\mathrm{rms}}$ at the trap. If we adjust the voltage to keep the stability parameter $q=0.25$, only $100 \mathrm{~mW} \mathrm{RF}$ input power is required at the modified resonance frequency.

We expect that one can improve the quality factor of the wire coil by using a thicker wire. We also tested a resonator with a lead-plated instead of the silver-plated copper wire, which did not show an increased voltage gain. Either we could not reach the critical temperature of lead in our test setup, or the lead was not pure enough to become superconducting.

The HTS spiral coil showed the highest voltage gain, although the shield influences the magnetic field generated by the coil. We observed a change of the resonance frequency of a couple of percent when cooling down from liquid nitrogen temperatures to liquid helium temperatures, which we attribute to the shield surrounding the coil. During this cooldown, the losses in the resonator dropped by about $20 \%$ confirming that the resonator is not limited by skin effect in the gold layer on top of the HTS coil. With a voltage gain of 92, one would need about $70 \mathrm{~mW}$ of RF input power to get $170 V_{\text {rms. }}$. With a stability parameter $q=0.25$ at the higher resonance frequency, it would be $82 \mathrm{~mW}$.

These resonators can be compared to devices used in other experiments $[16,17,23]$. Although the voltage gain there seems comparable, one needs to keep in mind that due to (6), the voltage gain is inversely proportional to the frequency and the capacitive load. Taking this into consideration, the resonator with the HTS coils outperforms all other resonators listed in Table 1. But such comparison neglects that helical resonators like in references $[16,23]^{4}$ typically require more volume than RLC resonators.

\footnotetext{
4 The exact load capacitances were not mentioned in these publications but were assumed to be similar to our $10 \mathrm{pF}$.
}

\section{Conclusion}

We have demonstrated three designs of RF resonators for trapped ion experiments with Paul traps. The PCB coils have favorable mechanical properties but still need an improved production process, such as e.g. thermal annealing. The wire coils fulfill our criteria and can be further improved by using a thicker or superconducting wire. The HTS spiral coil showed the highest voltage gain, which was already accessible at temperatures reached with liquid nitrogen cooling.

Efficient matching is usually difficult in a cryogenic environment because normally one cannot tune the matching parameters when the cryostat is cold. We have shown a recipe to efficiently match RLC resonators without precise knowledge of their quality factors. During this empirical study, we regularly achieved matching with a power reflection of less than $2 \%$ on the second cooldown. Additionally, we designed and implemented a shield for resonators to minimize RF pickup in other parts of the experiment.

Acknowledgments We thank Gerhard Hendl from the Institute of Quantum Optics and Quantum Information of the Austrian Academy of Science for the production of PCBs and PCB coils for this study. Furthermore, we want to thank Florian Ong for feedback on the manuscript. This research was funded by the Office of the Director of National Intelligence (ODNI), Intelligence Advanced Research Projects Activity (IARPA), through the Army Research Office Grant W911NF-10-1-0284. All statements of fact, opinion, or conclusions contained herein are those of the authors and should not be construed as representing the official views or policies of IARPA, the ODNI, or the US Government.

Open Access This article is distributed under the terms of the Creative Commons Attribution 4.0 International License (http://creativecommons.org/licenses/by/4.0/), which permits unrestricted use, distribution, and reproduction in any medium, provided you give appropriate credit to the original author(s) and the source, provide a link to the Creative Commons license, and indicate if changes were made.

\section{References}

1. W. Paul, Rev. Mod. Phys. 62, 531 (1990)

2. M. Chwalla, J. Benhelm, K. Kim, G. Kirchmair, T. Monz, M. Riebe, P. Schindler, A.S. Villar, W. Haensel, C.F. Roos, R. Blatt, M. Abgrall, G. Santarelli, G.D. Rovera, Ph Laurent, Phys. Rev. Lett. 102, 023002 (2009)

3. H.S. Margolis, G. Huang, G.P. Barwood, S.N. Lea, H.A. Klein, W.R.C. Rowley, P. Gill, R.S. Windeler, Phys. Rev. A 67, 032501 (2003)

4. J.I. Cirac, P. Zoller, Phys. Rev. Lett. 74, 4091 (1995)

5. D. Kielpinski, C. Monroe, D.J. Wineland, Nature 417, 709 (2002)

6. T. Monz, P. Schindler, J.T. Barreiro, M. Chwalla, D. Nigg, W.A. Coish, M. Harlander, W. Hänsel, M. Hennrich, R. Blatt, Phys. Rev. Lett. 106, 130506 (2011)

7. D. Porras, J.I. Cirac, Phys. Rev. Lett. 92, 207901 (2004) 
8. P. Jurcevic, B.P. Lanyon, P. Hauke, C. Hempel, P. Zoller, R. Blatt, C.F. Roos, Nature 511, 202 (2014)

9. D.J. Wineland, C. Monroe, W.M. Itano, D. Leibfried, B.E. King, D.M. Meekhof, J. Res. Natl. Inst. Stand. Technol. 103, 259 (1998)

10. Q.A. Turchette, B.E. King, D. Leibfried, D.M. Meekhof, C.J. Myatt, M.A. Rowe, C.A. Sackett, C.S. Wood, W. Itano, C. Monroe, D.J. Wineland, Phys. Rev. A 61, 063418 (2000)

11. J. Labaziewicz, Y. Ge, P. Antohi, D. Leibrandt, K.R. Brown, I.L. Chuang, Phys Rev. Lett. 100, 013001 (2008)

12. W.W. Macalpine, R.O. Schildknecht, Proc. IRE 47, 2099 (1959)

13. J.D. Siverns, L.R. Simkins, S. Weidt, W.K. Hensinger, Appl. Phys. B 107, 921 (2012)

14. S.R. Jefferts, C. Monroe, E.W. Bell, D.J. Wineland, Phys. Rev. A 51, 3112 (1995)

15. R. Franz, G. Wiedemann, Ann. Phys. 165, 497 (1853)

16. M.E. Poitzsch, J.C. Bergquist, W.M. Itano, D.J. Wineland, Rev. Sci. Instrum. 67, 129 (1996)
17. D. Gandolfi, M. Niedermayr, M. Kumph, M. Brownnutt, R. Blatt, Rev. Sci. Instrum. 83, 084705 (2012)

18. W. Paul, O. Shelton, E. Fischer, Forschungsberichte des Wirtschafts- und Verkehrsministeriums NRW 415, 1 (1958)

19. J.W. Ekin, Experimental Techniques For Low-Temperature Measurements (Oxford University Press, Oxford, 2006), p. 504

20. P. Kamby, A. Knott, M.A.E. Andersen, in IECON 2012 - 38th Annual Conference on IEEE Industrial Electronics Society (2012) p. 680

21. S.S. Mohan, The design, modeling and optimization of on-chip inductor and transformer circuits (Ph.D. thesis, Department of Electrical Engineering, Stanford University, 1999)

22. D.M. Pozar, Microwave Engineering (Wiley, New York, 2011)

23. S.X. Wang, Quantum gates, sensors, and systems with trapped ions (Ph.D. thesis, Department of Physics, Massachusetts Institute of Technology, 2012) 